\title{
Matching of Siberian Snakes
}

\author{
Georg H. Hoffstaetter
}

Cornell University, Ithaca/NY

\begin{abstract}
It is shown how one can choose suitable combinations of Siberian Snakes and betatron phase advances to optimize the ability of a ring to accelerate a polarized beam with little reduction of polarization. In an analysis of HERA-p, these methods have lead to a 14-fold increase of the vertical beam emittance for which polarization could be preserved. This is not only impressive as a result, but also the methods which lead to this result are very interesting. They contain detailed spin-orbit tracking and the application of the Froissart-Stora Formula for higher order spin-orbit resonances, for which an algorithm of determining resonance strength has been found.
\end{abstract}

\section{INTRODUCTION}

In order to produce polarized proton or deuteron beams at high energy, a polarized source is used and the beam is accelerated from low energy with as little loss of polarization as possible. Subsequently, the beam has to be stored at high energy with little depolarization for a long time, e.g. for many millions of turns around a storage ring.

While the polarized beam travels along the azimuth $\theta$ of the circular accelerator, its spin motion is influenced by external fields. Ideally, the center of the beam would travel in a horizontal plane and would only experience a vertical magnetic field so that a vertical spin would not precess and would therefore remain vertical from turn to turn. But even with field perturbations, a particle which travels along a periodic orbit and defines the center of a beam has a spin $\vec{n}_{0}$ which is periodic from turn to turn. However, $\vec{n}_{0}$ is in general not vertical. Other particles of the beam oscillate around the closed orbit during their motion around the circular accelerator and experience additional electromagnetic fields. These oscillations in phase space are described by $\vec{z}(\theta)$. The spins of those particles precess around a vector which differs from the vector around which particles on the closed orbit precess. The components of this vector which are perpendicular to $\vec{n}_{0}$ are expressed in the complex plane as $\omega(\vec{z}(\theta), \theta)$. These components cause a rotation of spins away from $\vec{n}_{0}$ and therefore cause depolarization of the beam. The depolarization is enhanced when the spin precession frequency around $\vec{n}_{0}$, the so called closed orbit spin tune $v_{0}$, is in resonance with a Fourier component of $\omega(\vec{z}(\theta), \theta)$. The strength of this depolarizing effect is characterized by the first order resonance strength,

$$
\varepsilon_{\kappa}=\lim _{N \rightarrow \infty} \frac{1}{2 \pi N} \int_{0}^{2 \pi N} \omega(\vec{z}(\theta), \theta) e^{-i \kappa \theta} d \theta
$$

Since the components of the phase space vector oscillate with the transverse tunes $Q_{x}$, $Q_{y}$, and the synchrotron tune $Q_{\tau}$, resonances occur when the closed orbit spin tune $v_{0}$ is a linear combination of these orbital tunes, i.e. $v_{0}=j_{0}+\vec{j} \cdot \vec{Q}=\kappa$ where all components 
$j_{k}$ of $\vec{j}$ are integers. Often the spin motion is approximated by the single resonance model (SRM) where only the dominant Fourier component of $\omega(\vec{z}(\theta), \theta)$ is taken into account and all other Fourier components are neglected.

The spin motion in the SRM is simple enough to be solved analytically. If the closedorbit spin tune changes linearly, i.e. $d v_{0} / d \theta=\tilde{\alpha}$, and if the spin is initially choses as vertical, then the vertical spin component long after the resonance is crossed is given by the Froissart-Stora formula,

$$
s_{3}(\infty)=2 e^{-\pi \frac{\varepsilon_{\kappa}^{2}}{2 \tilde{\alpha}}}-1
$$

For slow crossing of the resonance, i.e. small $\tilde{\alpha}$, the spin is flipped from vertically up to vertically down and for very fast crossing of the resonance, the spin is hardly affected and remains vertically up.

The Froissart-Stora formula is regularly used to describe the reduction of polarization due to vertical betatron oscillations during resonance crossing in accelerators where the closed-orbit spin tune $v_{0}$ changes with energy. These descriptions were normally restricted to first order resonances, flat rings, and $v_{0}=G \gamma$. In principle, also higher-order resonances can be treated in the SRM and could then be described by the FroissartStora formula. However, it has not been clear how to obtain the strength of higher order resonances since they cannot be computed by equation (1). Due to the nonlinear character of spin rotations, higher-order resonances can appear even when $\omega$ has only first order Fourier coefficients as in the case of linearized orbit motion. In fact, all higher order resonances which will be presented here have been computed with an $\omega(\vec{z}, \theta)$ which is linear in $\vec{z}$.

Since orbit tunes cannot be close to 0.5 , Siberian Snakes which fix the closed orbit spin tune to 0.5 avoid first order resonances completely. Higher order resonances then become important, and in the following, it will be demonstrated how the influence of higher order resonances can be computed and minimized. It will even be shown that a Froissart-Stora like formula can be applied and therefore resonance strength can be computed quantitatively that characterize phenomena like spin flipping and depolarization at higher order resonances.

\section{Motion of Spin Fields and the Invariant Spin Field}

To achieve a more complete description of the depolarization process it is helpful to analyze not only the motion of individual spins but to investigate the dynamics of the spin field of a beam. A spin field $\vec{f}(\vec{z}, \theta)$ with $|\vec{f}|=1$ describes the polarization of the beam by determining that each particle with the phase space coordinate $\vec{z}$ has the polarization direction $\vec{f}(\vec{z}, \theta)$ while it travels along the ring's azimuth $\theta$. While each spin precesses around a vector $\vec{\Omega}(\vec{z}, \theta)$, the precession of the spin field is described by

$$
\frac{d}{d \theta} \vec{f}=\partial_{\theta} \vec{f}+\left[\vec{v}(\vec{z}, \theta) \cdot \partial_{\vec{z}}\right] \vec{f}=\vec{\Omega}(\vec{z}, \theta) \times \vec{f} .
$$

If all particles of a beam are initially completely polarized parallel to each other, the polarization state of the beam is in general not $2 \pi$-periodic and the beam polarization 
can change from turn to turn. A special spin field $\vec{n}(\vec{z}, \theta)$ which is $2 \pi$-periodic in $\theta$ is called an invariant spin field or Derbenev-Kondratenko $\vec{n}$-axis,

$$
\vec{n}(\vec{z}, \theta+2 \pi)=\vec{n}(\vec{z}, \theta) .
$$

If the spin of each particle in a beam is initially parallel to $\vec{n}(\vec{z}, \theta)$, particles get redistributed in phase space during one turn, but their spins will stay parallel to the invariant spin field. The spin field of the beam is then in an equilibrium state. Note that $\vec{n}(\vec{z})$ is usually not an eigenvector of the one turn spin transport matrix $\underline{R}(\vec{z})$ at some phase space point since the spin of a particle has changed after one turn around the ring, but the eigenvector would not have changed.

However, although it has been straightforward to define $\vec{n}(\vec{z}, \theta)$, it is not easy to calculate this spin field in general and much effort has been spent on this topic, mostly for electrons at energies up to $46 \mathrm{GeV}$. All algorithms developed before the polarized proton project at HERA-p $[1,2,3,4]$ rely on perturbation methods at some stage, and either do not go to high enough order $[5,6]$ or have problems with convergence at high order and high proton energies $[7,8,9]$.

It can be shown that no spin field $\vec{f}(\vec{z}, \theta)$ has a time averaged polarization that is larger than that of the invariant spin field, which is given by the phase space average $P_{\text {lim }}=\langle\vec{n}(\vec{z})\rangle_{\vec{z}}$. Furthermore, the invariant spin field is periodic from turn to turn and can therefore be used to define a basis to describe spin motion for particles which oscillate around the closed orbit due to their phase space amplitudes $\vec{J}$. The spin precessions in this coordinate system can be used to define an amplitude-dependent spin tune that gives rise to an amplitude dependent resonance condition, $v(\vec{J})=j_{0}+\vec{j} \cdot \vec{Q}$.

\section{The Froissart-Stora Formula for Higher-Order Resonances}

For the SRM the invariant spin field $\vec{n}$, the time average maximum polarization $P_{\text {lim }}$, and the amplitude-dependent spin tune $v(\vec{J})$ can be computed analytically, and are illustrated in Fig. 1. The spin tune is given by

$$
v=\operatorname{sig}(\delta) \sqrt{\delta^{2}+\varepsilon_{\kappa}^{2}}+\kappa,
$$

where $\kappa$ is the spin tune at which the resonance condition is satisfied and $\delta=v_{0}-\kappa$ is the distance of the closed orbit spin tune from the resonance. In this formula and in Fig. 1, it is apparent that the spin tune jumps by twice the resonance strength at the location where the closed orbit spin tune satisfies the resonance condition.

Before the investigations in [3], it was not known how resonance strengths of higher order could be computed since they cannot be computed by equation (1). But once one is able to compute the amplitude-dependent spin tune, there is however a simple method: compute the amplitude-dependent spin tune and observe its jump where the closed orbit spin tune satisfies a resonance condition. The jump is $2 \varepsilon_{\kappa}$.

In [3], it has been shown that a SRM for the amplitude dependent spin tune close to an isolated resonance can be derived. However, here we simply track a beam that is initially 


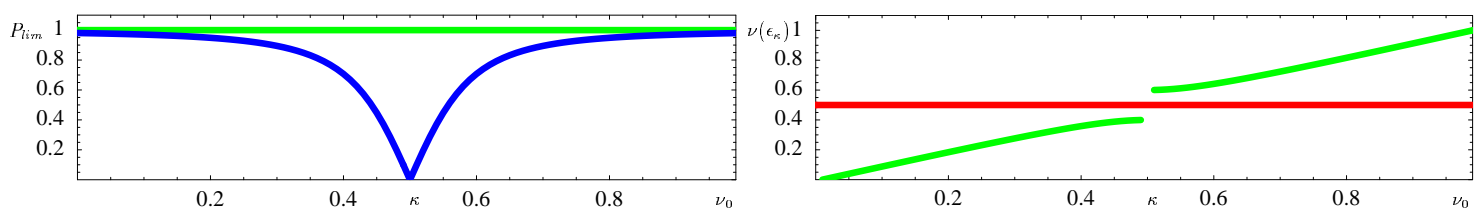

FIGURE 1. $P_{\text {lim }}$ and the amplitude-dependent spin tune $v\left(\varepsilon_{\kappa}\right)$ for the SRM in the vicinity of $v_{0}=\kappa$, for $\kappa=0.5$ and $\varepsilon_{\kappa}=0.1$.

polarized parallel to $\vec{n}(\vec{z})$ and accelerate it through a higher order resonance condition of the amplitude-dependent tune. If the remaining polarization in the direction of the new $\vec{n}$-axis at high energy follows the Froissart-Stora Formula, then we know that the strength of the higher order resonance has been determined.

In Fig. 2 (left), $P_{\text {lim }}$ and $v$ are shown after 4 Siberian Snakes were added to the simulation of the HERA-p optics used in 2002. $P_{\text {lim }}$ is reduced at two resonances with $v=2 Q_{y}$.

The spins of a set of particles were set parallel to the invariant spin field $\vec{n}(\vec{z})$ so that all had $J_{S}=\vec{n}(\vec{z}) \cdot \vec{S}=1$ at the momentum of $801 \mathrm{GeV} / \mathrm{c}$. The $\vec{n}$-axis had been computed by stroboscopic averaging [1]. The beam was then accelerated to $804 \mathrm{GeV} / \mathrm{c}$ at various rates of acceleration. The average $\bar{J}_{S}$ over the tracked particles after acceleration is plotted versus rate of acceleration in Fig. 2 (right) together with the prediction of the Froissart-Stora formula for which the resonance strength $\varepsilon_{2 Q_{y}}$ has been determined from the tune jump. The parameter $\tilde{\alpha}$ is proportional to the energy increase per turn $d_{E}$ and is determined from the tune slope $\frac{\Delta v}{\Delta E}$ in Fig. 2 (center) by the relation $\tilde{\alpha}=\frac{1}{2 \pi} \frac{\Delta v}{\Delta E} d_{E}$. The polarization obtained by accelerating particles through the second order resonance agrees remarkably well with the Froissart-Stora formula. For the slow acceleration of about $50 \mathrm{keV}$ per turn in HERA-p, the polarization would be completely reversed for particles with an amplitude of 0.75 sigma for the chosen scheme of four Siberian Snakes. This would lead to a net reduction of beam polarization, since the spins in the center of the beam are not reversed.

The two data points at the largest rate of acceleration are lower than predicted by the Froissart-Stora formula. One possible explanation is the following: at very large rates of acceleration, the resonance region is crossed so quickly that the spin motion is hardly disturbed. But when the invariant spin field $\vec{n}_{-}$before the resonance region is not parallel to the invariant spin field $\vec{n}_{+}$after the resonance region, then the spins which initially had $J_{S}=\vec{n}(\vec{z}) \cdot \vec{S}=1$ will approximately have $J_{S}=\vec{n}_{-} \cdot \vec{n}_{+}$after the resonance region is crossed. This is smaller than the Froissart-Stora prediction, which approaches 1 for large rate of acceleration.

\section{SNAKE MATCHING}

In order to minimize depolarizing resonance effects, firstly Siberian Snakes are inserted in the ring to fix the closed orbit spin to 0.5, which avoids first order resonances of the closed orbit spin tune. In Fig. 3 (left), the energy dependences of $P_{\text {lim }}$ with and without 

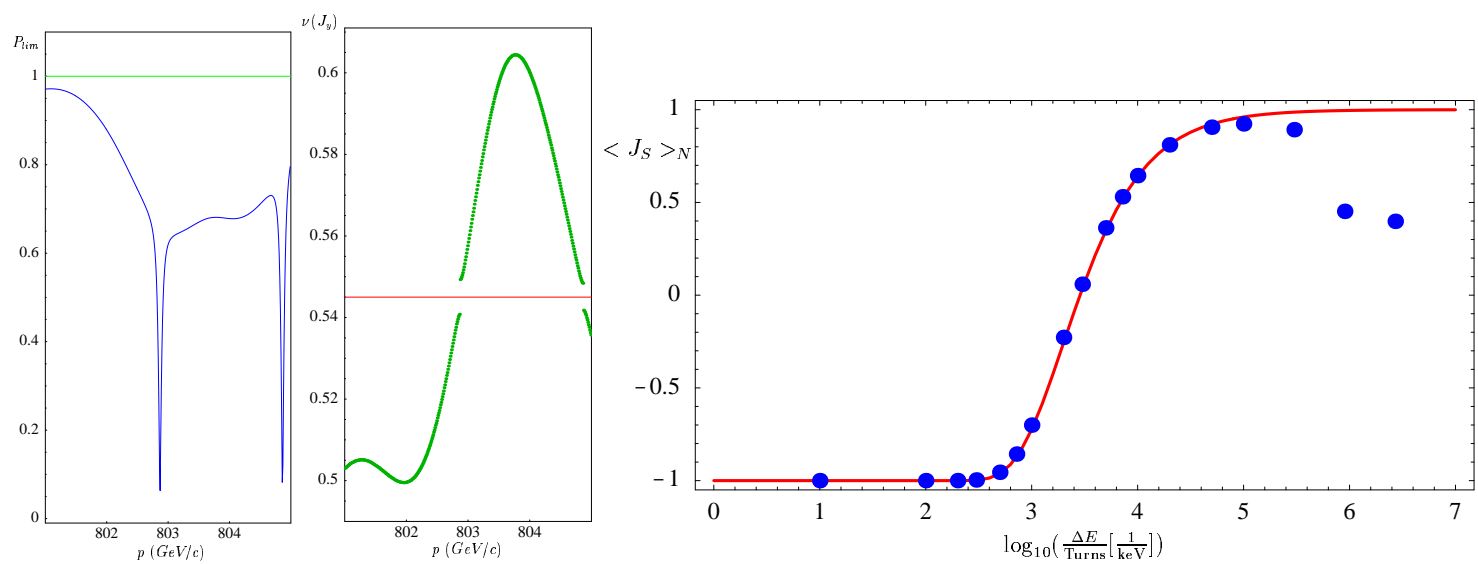

FIGURE 2. Left: $P_{\text {lim }}$ and $v$ for a second-order resonance after an addition of a 4-snake scheme to the HERA-p optics that was used in 2002 with a $0.75 \sigma$ vertical amplitude of $2.25 \pi$ mm mrad. Right: The average $\left\langle J_{S}\right\rangle_{N}$ over $N$ particles after acceleration from $801 \mathrm{GeV} / \mathrm{c}$ to $804 \mathrm{GeV} / \mathrm{c}$ with different rates of acceleration (points) and the prediction of the Froissart-Stora formula (curve) using parameters $\varepsilon_{2 Q_{y}}$ and $\tilde{\alpha}$ obtained from $v$ in the center plot.

Siberian Snakes are overlaid, which shows that not all resonance effects are removed by Siberian Snakes. In the energy regions where Siberian Snakes do not avoid the reduction of $P_{\text {lim }}$, the perturbations to spin motion in each FODO cell of HERA-p add up so that the resonance strengths are very large.

When designing a circular accelerator for polarized beams, one should therefore not only insert Siberian Snakes but also minimize the divergence or spread of the $\vec{n}$-axis, and this maximizes $P_{\text {lim }}$. Since the spin tune jump at higher order resonances relates to their strength, one should additionally try to minimize these jumps. If possible one should avoid that the spin tune crosses a low order resonance line. Figure 3 (right) shows the amplitude-dependent spin tune.
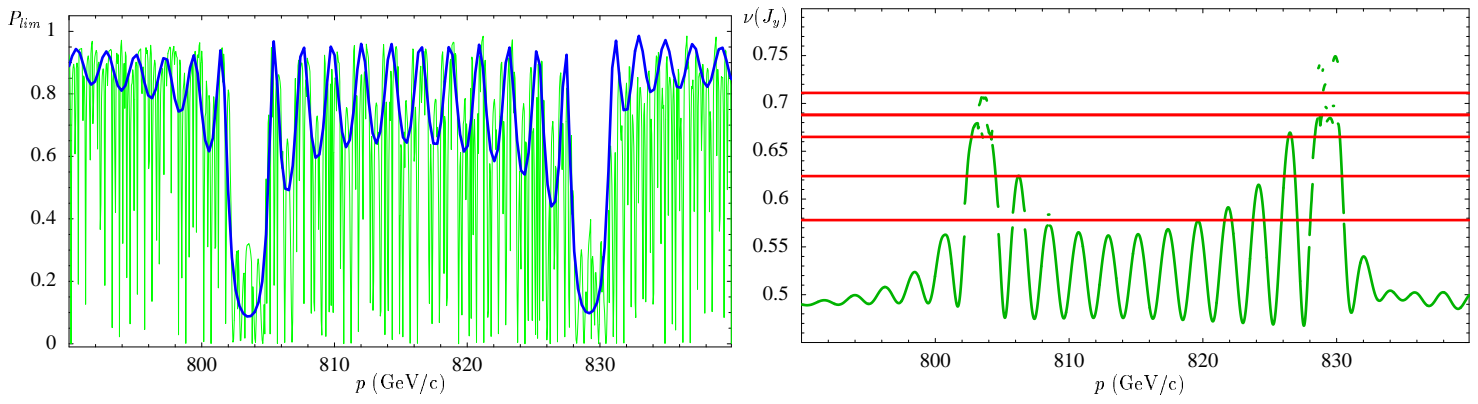

FIGURE 3. Left: First order $P_{\text {lim }}$ with and without Siberian Snakes. Right: The amplitude-dependent but orbital-phase-independent spin tune $v$ for four Siberian Snakes in HERA-p. From top to bottom, the following resonance lines are drawn: $v=1-Q_{y}, v=3-8 Q_{y}, v=5-15 Q_{y}, v=16 Q_{y}-4, v=2 Q_{y}$. The strength of these higher-order resonances can be deduced from the tune jumps.

Snake matching is the procedure proposed here for minimizing the spread of the $\vec{n}$-axis by minimizing the spin orbit coupling integrals. At azimuth $\theta_{0}$, the spin orbit 
coupling integrals are defined as

$$
I_{k}^{ \pm}=-i \int_{\theta_{0}}^{\theta_{0}+2 \pi} \omega\left(\vec{v}_{k}^{ \pm}, \theta\right) e^{i\left\{ \pm Q_{k}\left(\theta-\theta_{0}\right)-\Psi\right\}} d \theta
$$

where $\vec{v}_{k}^{ \pm}$are the eigenvectors of the one turn beam transport matrix and $\omega$ describes the spin rotation as in equation (1) for linearized phase space motion. The spin phase advance from $\theta_{0}$ to $\theta$ is $\Psi$, and $Q_{k}\left(\theta-\theta_{0}\right)$ is the orbit phase advance associated with the eigenmode $\vec{v}_{k}^{ \pm}$. When the spin-orbit-coupling integrals for $\theta_{0}$ are minimized, the opening angle of the invariant spin field at $\theta_{0}$ for the approximation of linear spin-orbit motion is also minimized [3].

The vertical spin-orbit-coupling integrals from the first regular FODO cell to the last FODO cell of a regular arc in HERA-p will be denoted by $\hat{I}_{y}^{+}$and $\hat{I}_{y}^{-}$and the azimuths of the beginnings of the 4 regular arcs are $\theta_{1}, \theta_{2}, \theta_{3}$, and $\theta_{4}$ as shown in Fig. 4 . The central points of the South, West, North, and East straight sections are denoted by $S, W$, $N$, and $E$. The spin phase advances between the arcs are suitably manipulated by the snake angles $\varphi_{E}, \varphi_{N}$, and $\varphi_{W}$. The closed orbit spin tune is then adjusted to 0.5 by $\varphi_{S}$. A snake angle $\phi$, i.e. the angle that a Siberian Snake's horizontal rotation axis makes to the radial direction, is important here since a Siberian Snake not only flips the spin by $180^{\circ}$, but also causes a spin phase advance of $2 \phi$ around the vertical. The spin phase advance between $\theta_{i}$ and $\theta_{j}$ is denoted by $\Psi_{i j}$. Figure 4 (left) also shows eight flattening snakes, one in each straight section. They compensate the non-flat regions of HERA-p.
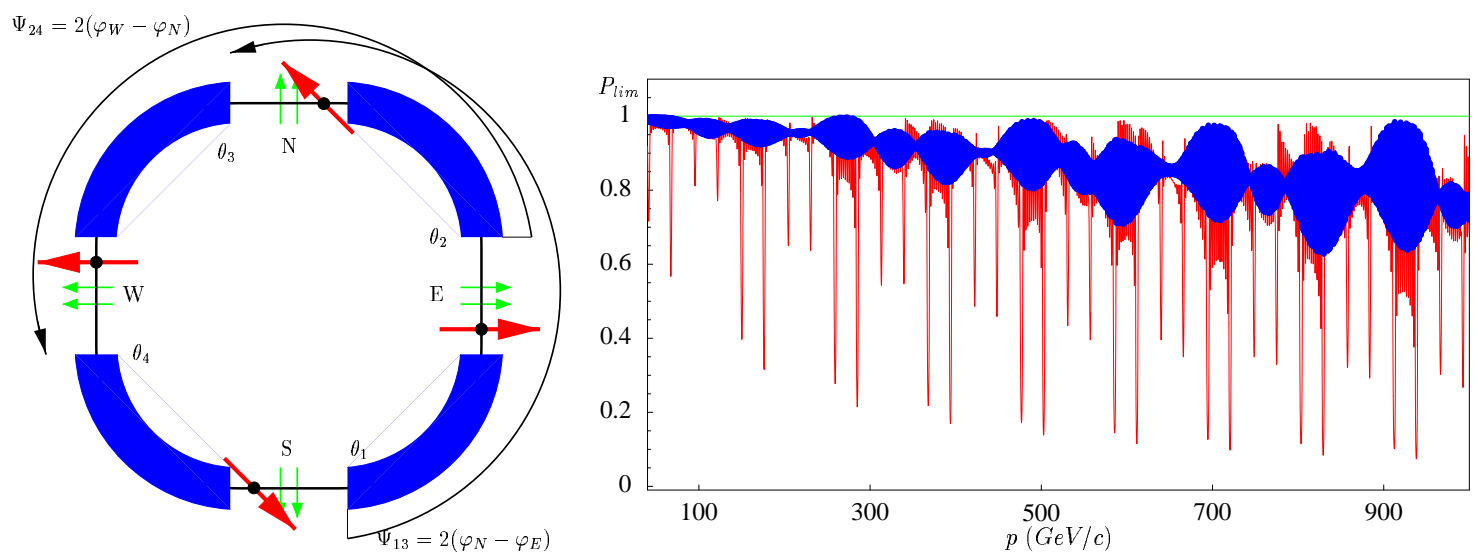

FIGURE 4. Left: The spin phase advance from the beginning of one regular arc to the beginning of the regular arc on the opposite side of the ring. Right: Improvement of linearized $P_{\text {lim }}$ by matching 4 snake angles and the orbital phases. The snake arrangement is $\left(0 \frac{\pi}{2} \frac{\pi}{2} \frac{\pi}{2}\right)^{*}$ (dark foreground curve). As a comparison, $P_{\text {lim }}$ from linearized spin-orbit motion is shown for the same HERA-p optics with a $\left(\frac{\pi}{4} 0 \frac{\pi}{4} 0\right)$ snake scheme (light background curve).

With these 8 flattening snakes and one Siberian Snake in each of the straight sections, the spin phase advance from $\theta_{1}$ to $\theta_{3}$ is given by $\Psi_{13}=\Psi_{1 E}-2 \varphi_{E}-\Psi_{E N}+2 \varphi_{N}+\Psi_{N 3}$. Here, the fact that a Siberian Snake rotates the coordinate system for spins was used, so that the spin phase in the spin orbit coupling integral changes its sign. The terms due to $\Psi$ cancel and the total spin phase advance is solely determined by the snake angles and is therefore independent of energy: $\Psi_{13}=2\left(\varphi_{N}-\varphi_{E}\right)$ and $\Psi_{24}=2\left(\varphi_{W}-\varphi_{N}\right)$. The 
orbital phase advance $\Phi_{y}\left(\theta_{3}\right)-\Phi_{y}\left(\theta_{1}\right)$ also does not depend on energy. For simplicity, $\Phi_{y}\left(\theta_{j}\right)-\Phi_{y}\left(\theta_{i}\right)$ will now be denoted by $\Phi_{i j}$.

The spin-orbit-coupling integrals at the South interaction point contain the following contributions from the 4 regular arcs:

$$
\begin{aligned}
I_{\mathrm{arcs}}^{+}= & \hat{I}^{+} e^{i\left(-\Psi_{S 1}+\Phi_{S 1}\right)}\left(1+e^{i\left[2\left(\varphi_{E}-\varphi_{N}\right)+\Phi_{13}\right]}\right) \\
& +\left(\hat{I}^{-}\right)^{*} e^{i\left(2 \varphi_{E}-\Psi_{S E}+\Psi_{E 2}+\Phi_{S 2}\right)}\left(1+e^{i\left[2\left(\varphi_{W}-\varphi_{N}\right)+\Phi_{24}\right]}\right), \\
I_{\mathrm{arcs}}^{-}= & \hat{I}^{-} e^{i\left(-\Psi_{S 1}-\Phi_{S 1}\right)}\left(1+e^{i\left[2\left(\varphi_{E}-\varphi_{N}\right)-\Phi_{13}\right]}\right) \\
& +\left(\hat{I}^{+}\right)^{*} e^{i\left(2 \varphi_{E}-\Psi_{S E}+\Psi_{E 2}-\Phi_{S 2}\right)}\left(1+e^{i\left[2\left(\varphi_{W}-\varphi_{N}\right)-\Phi_{24}\right]}\right) .
\end{aligned}
$$

This shows that it is always possible to cancel one of the spin-orbit coupling integrals by choosing the snake angles so that the spin perturbation produced in one of the arcs is canceled by the arc on the opposite side of the ring. Since $\left|\hat{I}^{+}\right|$and $\left|\hat{I}^{-}\right|$are different, neighboring arcs can in general not compensate each other.

To cancel both spin-orbit integrals in (8), 4 phase factors have to be -1 . This requires

$$
\begin{aligned}
2\left(\varphi_{E}-\varphi_{N}\right)+\Phi_{13} & =\pi \bmod 2 \pi, \\
2\left(\varphi_{E}-\varphi_{N}\right)-\Phi_{13} & =\pi \bmod 2 \pi, \\
2\left(\varphi_{W}-\varphi_{N}\right)+\Phi_{24} & =\pi \bmod 2 \pi, \\
2\left(\varphi_{W}-\varphi_{N}\right)-\Phi_{24} & =\pi \bmod 2 \pi .
\end{aligned}
$$

For arbitrary betatron phase advances, this equation cannot be solved by a choice of snake angles, since there are only two free parameters which contain the snake angles. However, the betatron phase advances can be changed appropriately. Subtraction of the first two equations leads to the requirement that the betatron phase advance from $\theta_{1}$ half way around the ring to $\theta_{3}$ be an odd or even multiple of $\pi$. The same is true for the phase advance from $\theta_{2}$ to $\theta_{4}$. Correspondingly, the spin phase advance over these regions has to be an odd multiple of $\pi$ when the orbit phase advance is an even multiple and vice versa. With a rather benign change of the vertical optics in HERA-p which does not change the vertical tune, the contribution of the regular arcs to both spin-orbit-coupling integrals can thus be canceled when 4 Siberian Snakes are in HERA-p.

In the following, we characterize snake schemes by their snake angles starting in the South: e.g. $\left(\phi_{S} \phi_{E} \phi_{N} \phi_{W}\right)$. The snake scheme $\left(0 \frac{\pi}{2} \frac{\pi}{2} \frac{\pi}{2}\right)^{*}$ has $\Psi_{13}=0$ and $\Psi_{24}=0$. The star indicates that the betatron phase advance has also been used for snake matching. For this snake scheme, the betatron phase advances from $\theta_{1}$ to $\theta_{3}$ and from $\theta_{2}$ to $\theta_{4}$ were adjusted to be odd multiples of $\pi$. The maximum time average polarization $P_{\text {lim }}$ for linearized spin-orbit motion is plotted (dark foreground curve) in Fig. 4 (right) for the complete range of HERA-p momenta. As a comparison, $P_{\text {lim }}$ for a standard snake scheme $\left(\frac{\pi}{4} 0 \frac{\pi}{4} 0\right)$ (light background curve) is also shown. The latter scheme and similar symmetric schemes were originally considered advantageous by a popular opinion [10], mostly due to their symmetry.

For linearized spin-orbit motion, the complete snake match of the arcs in HERA-p indeed eliminates all strong reductions of $P_{\text {lim }}$ over the complete momentum range. 


\section{Schemes with 8 snakes:}

Although eight snakes are not very practical for HERA-p, significant improvements are in principle possible when 8 snakes are used. A similar, only slightly more elaborate analysis shows that a choice of 8 snakes in the scheme characterized as $\left(\frac{\pi}{2} 0000000\right)^{*}$ can cancel the contributions of the regular arc part of individual octants against each other if betatron phase advances are chosen appropriately. In this snake scheme, $\Phi_{12}$ was changed to $2 \pi \times 8.5$ and $\Phi_{34}$ was changed to $2 \pi \times 7.5$ without changing the vertical tune.

There is a second scheme with 8 snakes which also cancels the contribution of individual octants. This snake scheme is referred to as $\left(\frac{\pi}{2} a b c 0-c-b-a\right)^{*}$.

\section{Nonlinear Spin Dynamics for Vertical Particle Motion}

To check whether the improvements of spin motion, obtained in the framework of linearized spin-orbit motion, survive when higher-order effects are considered, $P_{\text {lim }}$ and $v$ has been calculated by the SODOM-2 algorithm with the code SPRINT [11]. The result for the Siberian Snake scheme $\left(\frac{\pi}{4} 0 \frac{\pi}{4} 0\right)$, which was one of those that used to be considered advantageous by a popular opinion, is shown for the South interaction point of HERA-p in Fig. 5. Here many higher-order resonances are revealed, causing strong reduction of $P_{\text {lim }}$ and there are corresponding strong variations of the amplitudedependent spin tune $v$. The strongest spin tune jumps occur in the critical energy regions, mostly at the second order resonance $v=2 Q_{y}$, which is indicated by the top line [12].
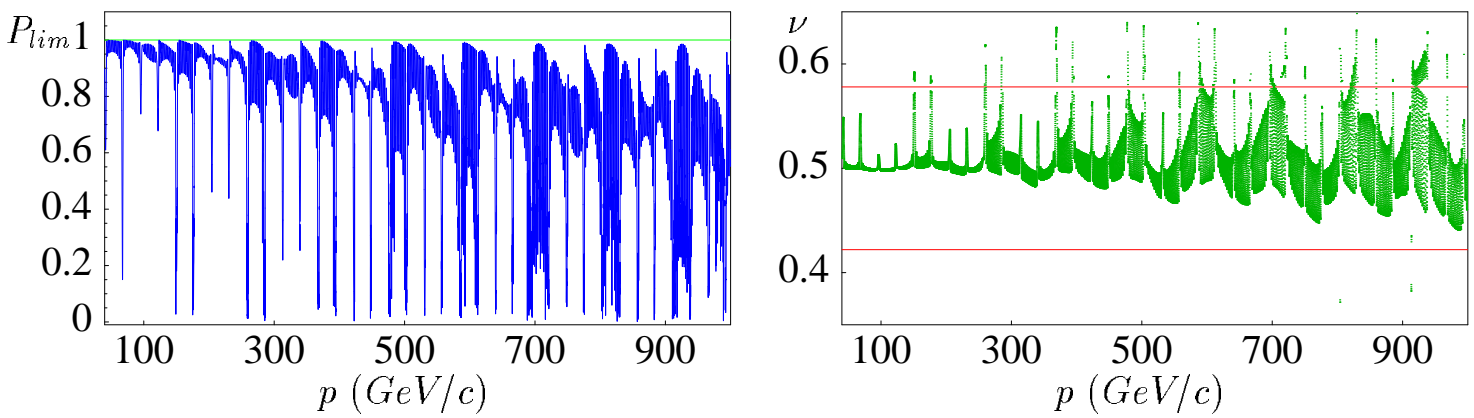

FIGURE 5. $P_{\text {lim }}$ and $v$ for particles with a $2.5 \sigma$ vertical amplitude in the HERA-p lattice of the year 2002 with the standard scheme $\left(\frac{\pi}{4} 0 \frac{\pi}{4} 0\right)$. The second-order resonances $v=2 Q_{y}$ and $v=1-2 Q_{y}$ are indicated by the two lines in the right graph.

$P_{\text {lim }}$ and $v$ for higher-order spin dynamics in the snake-matched and phase-advancematched HERA-p with the snake scheme $\left(0 \frac{\pi}{2} \frac{\pi}{2} \frac{\pi}{2}\right)^{*}$ are shown in Fig. 6. While the overall behavior of $P_{\text {lim }}$ over the complete acceleration range of HERA-p looks similar to the result obtained with linearized spin-orbit motion, which was displayed in Fig. 4 (right), higher-order effects become strong at high energies. But the spin tune spread at momenta below $400 \mathrm{GeV} / \mathrm{c}$ is small, and higher-order effects seem to be benign. The advantage over the snake scheme $\left(\frac{\pi}{4} 0 \frac{\pi}{4} 0\right)$ becomes clear: $v$ comes close to a second order resonance at much fewer places and only exhibit spin tune jumps which are much weaker than those shown in Fig. 5. 

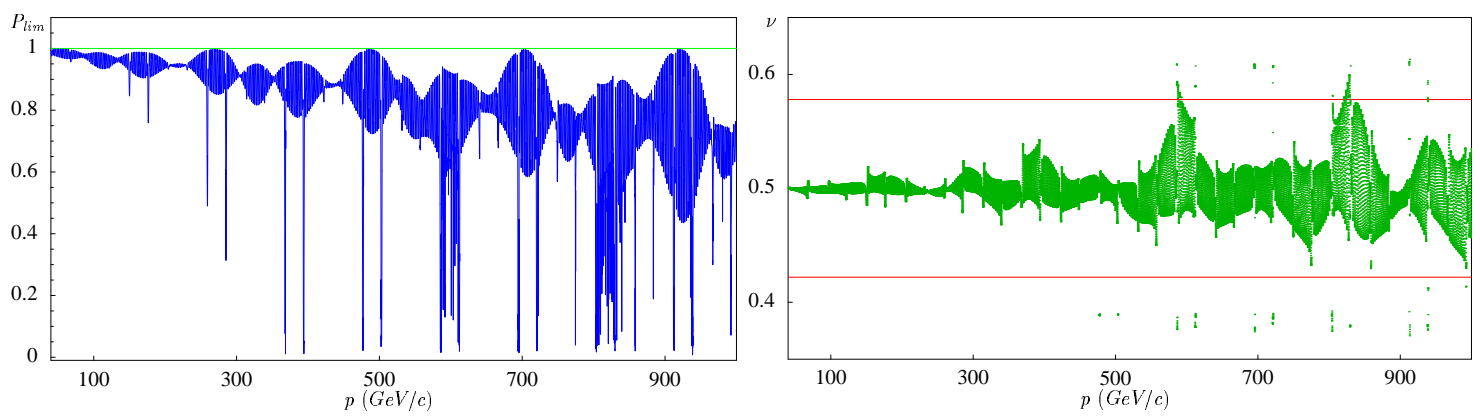

FIGURE 6. $\quad P_{\text {lim }}$ and $v$ for a $2.5 \sigma$ vertical amplitude after the betatron phase advance between opposite regular arc structures was adjusted to be an odd multiple of $\pi$ in the $\left(0 \frac{\pi}{2} \frac{\pi}{2} \frac{\pi}{2}\right)^{*}$ scheme. The resonances $v=2 Q_{y}$ and $v=1-2 Q_{y}$ are indicated by the two lines in the right graph.

\section{Allowed Beam Sizes}

After this optimization of snake schemes and the betatron phase advance, one finally has to check if, indeed, less depolarization occurs when particles are accelerated across the critical momentum region from 800 to $806 \mathrm{GeV} / \mathrm{c}$ with a typical rate of acceleration of $50 \mathrm{keV}$ per turn. For that, one distributes particles in phase space and starts spins parallel to the invariant spin field $\vec{n}(\vec{z})$. After the acceleration, one then checks whether the spins are still parallel to the new invariant spin field at the increased energy. The average projection of the spins onto the new $\vec{n}$-axis, $\bar{J}_{S}=\langle\vec{n} \cdot \vec{S}\rangle$ is shown in Fig. 7 (left) for different vertical oscillation amplitudes and for different snake schemes.

For the standard snake scheme $\left(\frac{\pi}{4} 0 \frac{\pi}{4} 0\right)$, only the part of the beam with less than $1 \pi \mathrm{mm}$ mrad vertical amplitude can remain polarized. For the scheme $\left(\frac{3 \pi}{4} \frac{3 \pi}{8} \frac{3 \pi}{8} \frac{\pi}{4}\right)$, which had been found by trial and error, phase space amplitudes up to $4 \pi \mathrm{mm}$ mrad are allowed. Finally, the snake matched scheme $\left(0 \frac{\pi}{2} \frac{\pi}{2} \frac{\pi}{2}\right)^{*}$ gives the most stable spin motion and Fig. 7 (left) shows that vertical amplitudes of up to $8 \pi \mathrm{mm}$ mrad are allowed.

The two different snake matches for 8 -snake schemes have also been tested. In Fig. 7 (right), it is shown that both lead to very small spin tune variations even compared to the matched 4-snake scheme. As shown in Fig. 7 (left), the more effective of the two 8 -snake schemes stabilizes spin motion up to a vertical amplitude of $14 \pi \mathrm{mm}$ mrad.

These results show that it is not possible to give a simple formula for the number of snakes which are required for a given accelerator since different snake schemes with the same number of snakes lead to very different stability of spin motion since the required number of snakes depends strongly on the chosen snake angles.

\section{ACKNOWLEDGMENTS}

A collaboration with Desmond Barber, Klaus Heinemann, Mathias Vogt, and Kaoru Yokoya has been very valuable in establishing the results presented here. Associating the gaps of the amplitude-dependent spin tune with resonance strength was inspired in 1999 by a comment of A. Lehrach during a talk by M. Vogt. 

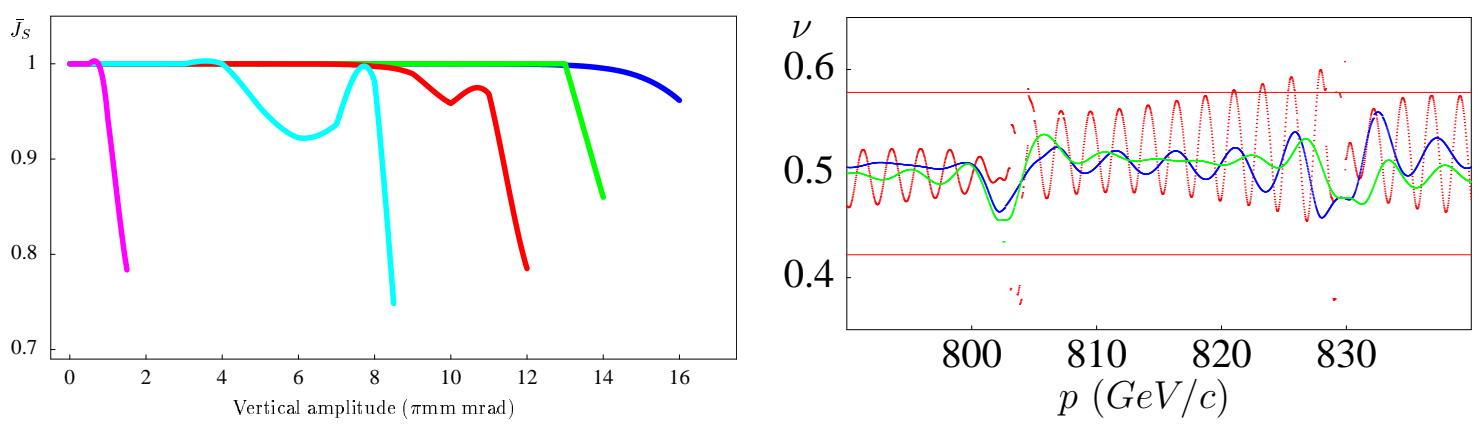

FIGURE 7. Left: The average spin action $\bar{J}_{S}$ at $806 \mathrm{GeV}$ for particles which started initially with $J_{S}=\vec{n} \cdot \vec{S}=1$ at $800 \mathrm{GeV} / \mathrm{c}$. From the leftmost to the rightmost curve: 1) The standard scheme which stabilizes spin motion for particles within $1 \pi \mathrm{mm}$ mrad, 2) a 4-snake scheme found by trial and error which stabilizes within $4 \pi \mathrm{mm} \mathrm{mrad}, 3$ ) the matched 4-snake scheme which stabilizes within $8 \pi \mathrm{mm} \mathrm{mrad}, 4$ ) the matched 8-snake scheme which stabilizes within $13 \pi \mathrm{mm} \mathrm{mrad}, 5)$ and the matched 8-snake scheme which stabilizes within $14 \pi \mathrm{mm}$ mrad. Right: Improvement of $v\left(J_{y}\right)$ by matching 8 snake angles and the orbital phases. The snake arrangements are $\left(\frac{\pi}{2} 0000000\right)^{*}($ dark $),\left(\frac{\pi}{2} a b c 0-c-b-a\right)^{*}($ light $)$, and $\left(0 \frac{\pi}{2} \frac{\pi}{2} \frac{\pi}{2}\right)^{*}$ (curve with largest oscillations). The resonances $v=2 Q_{y}$ and $v=1-2 Q_{y}$ are indicated by lines.

\section{REFERENCES}

1. K. Heinemann and G. H. Hoffstaetter, A tracking algorithm for the stable spin polarization field in storage rings using stroboscopic averaging, Phys. Rev. E 54, 4240-4255 (1996).

2. G. H. Hoffstaetter, M. Vogt, and D. P. Barber, Higher-order effects in polarized proton dynamics, Phys. Rev. ST-AB, 2(11), 1-15 (1999).

3. G. H. Hoffstaetter, Aspects of the invariant spin field for high energy polarized proton beams, habilitation, Darmstadt Univ. of Tech. (January 2000).

4. M. Vogt, Bounds on the maximum attainable equilibrium spin polarization of protons in HERA, Dissertation, Universität Hamburg, DESY-THESIS-2000-054 (December 2000).

5. A. W. Chao, Evaluation of radiative spin polarization in an electron storage ring, Nucl. Instrum. Methods 180, 29 (1981).

6. Yu. Eidelman and V. Yakimenko, The application of Lie methods to the spin motion in nonlinear collider fields, Particle Accelerators, 45, 17-35 (1994).

7. S. R. Mane, Electron-spin polarization in high-energy storage rings II. Evaluation of the equilibrium polarization, Phys. Rev. A(36), 120-130 (1987).

8. K. Yokoya, Non-perturbative calculation of equilibrium polarization of stored electron beams, Report KEK-92-6, Tsukuba (1992).

9. V. Balandin and N. Golubeva, Nonlinear spin dynamics, Proceedings of the XV International Conference on High-energy Particle Accelerators, Hamburg, pp. 998-1000 (1992).

10. V. Ptitsin and Yu. M. Shatunov, The investigation of spin resonances in an accelerator with Siberian Snakes, in C. W. de Jager, T. J. Ketel, P. J. Mulders, J. E. J. Oberski, and M. Oskam-Tamoezer, editors, Proceedings SPIN96, pp. 516-518, World Scientific (1996).

11. K. Yokoya, An algorithm for calculating the spin tune in circular accelerators, Report DESY-99-006 (1999).

12. D. P. Barber, G. H. Hoffstaetter, and M. Vogt, Using the amplitude-dependent spin tune to study high order spin-orbit resonances in storage rings, AIP Conf. Proc. of the 14th intl. Spin Physics Symposium, SPIN2000, 570, pp. 751-755 (2001). 